“C 2019 IEEE. Personal use of this material is permitted. Permission from IEEE must be obtained for all other uses, in any current or future media, including reprinting/republishing this material for advertising or promotional purposes, creating new collective works, for resale or redistribution to servers or lists, or reuse of any copyrighted component of this work in other works." 


\title{
A Thermal Management System to Reuse Thermal Waste Released by High-Power Light-Emitting Diodes (HP-LEDs)
}

\author{
Ning Wang, Member, IEEE, Cong Gao, Can Ding, Hong-Zhi Jia, Guo-Rong Sui, and Xiu-Min Gao
}

\begin{abstract}
In this paper, a comprehensive and efficient thermal management system is proposed to harvest and reuse the thermal waste of high-power light-emitting diodes (HP-LEDs) for the first time. Besides a conventional cooling system including a thermoelectric cooler (TEC), a heatsink, and a fan, the proposed thermal management system also employs a thermoelectric generator (TEG), a temperature sensor, a voltage boost converter, and a microcontroller for thermal waste recycling. In this system, some of the thermal waste released by the HP-LED is harvested by the TEG and converted to electrical energy. With the help of a voltage boost converter, the harvested electrical power is used to power a temperature sensor to monitoring the surface temperature of the HP-LED. The entire system is regulated by the microcontroller. The system is elaborately established, tested, and the results are discussed. The experimental results show that the proposed system has an output electrical power of approximately 696.5 $\mu \mathrm{W}$, which is used to power a temperature sensor as a demonstration. The sensor works well and the discrepancy of the surface temperature of the HP-LED measured by the sensor and by a thermometer is less than $5.38 \%$, which validates the proposed thermal management system.
\end{abstract}

Index Terms-Energy harvesting, energy recycling, environmental monitoring, high-power light-emitting diodes (HP-LEDs), thermoelectric generator (TEG), thermal management, thermal waste.

\section{INTRODUCTION}

$\mathrm{H}$ IGH-POWER light-emitting diodes (HP-LEDs) have been widely used in modern lighting due to their long service life, fast response speed, and energy saving characteristics. However, the electro-optic conversion efficiency of HP-LEDs is still low, i.e., approximately $20 \%$ to $40 \%$ [1]-[4]. More than two-thirds of the electrical energy consumed by the HP-LED is released in the form of heat, affecting the performance of the

Manuscript received; This work was supported by National key research and development plan "earth observation and navigation" key special project (2017YFB0503102), National Key Research and Development Program of China (2018YFC1313803), and NSFC (61804096). (Corresponding author: Can Ding.)

N. Wang, C. Gao, H.-Z. Jia, G.-R. Sui, and X.-M. Gao are with the Engineering Research Center of Optical Instrument and System, Ministry of Education, Shanghai Key Lab of Modern Optical System, University of Shanghai for Science and Technology, Shanghai, China (e-mail: nwang@usst.edu.cn).

C. Ding is with the Global Big Data Technologies Center, University of Technology Sydney, Sydney, NSW 2007, Australia (e-mail: can.ding.1989@gmail.com). diode [5], [6]. To avoid the reduction of luminosity and change in wavelength emission of HP-LEDs [7], [8], the junction temperature should not exceed an upper limitation [9]. This requires an efficient heat dissipation to reduce the junction temperature [10].

Heat dissipation of LED can be improved by employing efficient cooling devices to rapidly transfer the released heat from the LED base to external environment [11]. One promising cooling device is thermoelectric cooler (TEC) [12]-[15], which has been successfully used for heat dissipation of HP-LEDs. For example, in [16], an automatic temperature control system was proposed, consisting of three parts, i.e., a TEC, a microfan, and a microcontroller. When the temperature of the LED reaches $43^{\circ} \mathrm{C}$, this cooling system starts to work and the temperature will soon be reduced to a pre-set value of $30^{\circ} \mathrm{C}$. Yuruker et al. [17] studied the thermal and optical performances of LED under different driving conditions and used a TEC to control the board temperature. Although there exist several approaches to control the temperature of HP-LED, no effort has been made to handle the substantial thermal waste released from HP-LEDs. In this paper, we propose to reuse this wasted thermal energy to power low-power electronics.

Thermal energy can be harvested by a combination of a thermoelectric generator (TEG) and a DC-to-DC converter [18]. The former converts heat flux into electrical energy and the latter converts a source of direct current (DC) from one voltage level to another. Using this method, even a small temperature difference of only $4^{\circ} \mathrm{C}$ can generate enough electrical energy for some low-power electronic devices. Specifically, thermal energy harvesting is the process of converting thermal gradients into electrical energy through TEG [19]. For example, Wang et al. [20] demonstrated a novel wearable TEG device that uses human body heat to power a miniaturized accelerometer. Experiment shows that the proposed TEG provides a stable long-term power for an accelerometer to detect human motion. Furthermore, there are also examples of system-on-chips (SoC) [21], autonomous multisensor systems [22], and wireless sensors [23] being successfully powered by diverse energy harvesting systems based on TEGs. In these cases, there are various heat sources, such as human body heat, solar radiation, and high-temperature fluid pipelines. However, to a certain extent, these systems are constrained by environment and location due to the 
characteristics of the heat sources; thus, the output power of these systems is unsustainable and not continuous.

HP-LED is a stable and sustainable heat source. In this work, we propose to use the temperature gradient generated by a working HP-LED as the input of TEG to generate electrical power. Different from conventional thermal management systems focusing on efficient heat dissipation, this work aims to enable an optimal use of the waste heat and a comprehensive control of the thermal power harvesting. In this paper, a thermal management system is developed integrating both a thermoelectric cooling module and a thermal energy harvesting module. As a demonstration, the energy harvested from the HP-LED is used to power a temperature sensor monitoring the temperature of the HP-LED, which is crucial to LED operation. This is the first time that the waste heat released by a HP-LED is recycled and utilized to power low-power electronics.

The rest of the paper is organized as follows. The thermoelectric properties of the TEG employed in this work are theoretically analyzed and experimentally characterized in Section II. Section III presents the design flow of the system. In Section IV, the performance of the proposed thermal management system is evaluated, and the experimental results are discussed. Finally, this paper is concluded in Section V.

\section{Performance Characterization OF TEG}

\section{A. Theoretical analysis of the thermoelectric properties}

As illustrated in Fig. 1(a), a typical single-stage TEG consists of an array of thermoelectric legs (TE legs) sandwiched between two thermally conducting but electrically insulating ceramic plates. The TE legs are electrically connected in series by copper connectors. When exposed to a temperature gradient, i.e., the temperature of one side of the TEG is higher than that of the other side, an electromotive force will be induced across the thermocouples. Fig. 1(b) shows the electrical equivalent circuit of the TEG. The resultant open-circuit voltage across the TEG terminals $V_{o c}$ can be expressed by
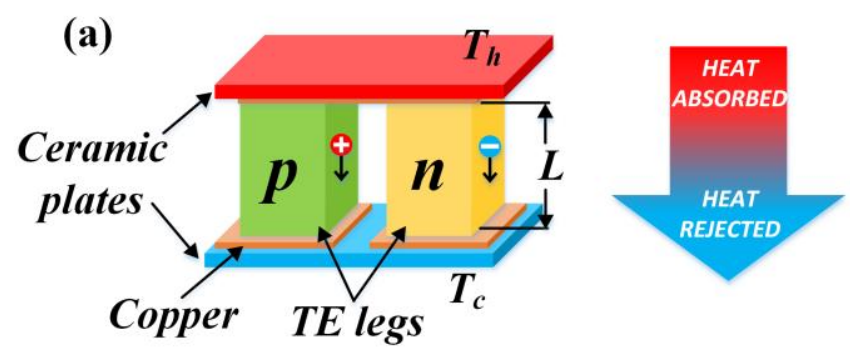

(b)

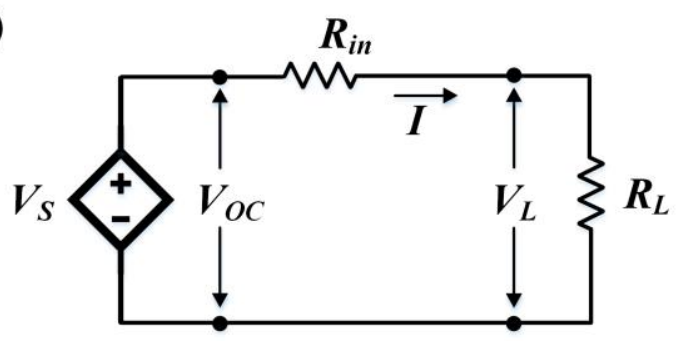

Fig. 1. (a) Schematic diagram and (b) equivalent circuit of a typical TEG module.

$$
V_{O C}=V_{S}=S \times\left(T_{h}-T_{c}\right)=S \times \Delta T,
$$

where $V_{S}$ is the Seebeck electromotive force, $T_{h}$ and $T_{c}$ are the hot- and cold-side temperatures, $\Delta T$ is the temperature difference across the junctions, and $S$ is the Seebeck coefficient of the module. According to [24], $S$ can be obtained as

$$
S=N\left(\alpha_{p}-\alpha_{n}\right)
$$

where $\alpha_{n}$ and $\alpha_{p}$ are the absolute Seebeck coefficients for the n-type and p-type materials of the thermoelectric legs, respectively, and $N$ is the total number of thermocouples.

Moreover, the total resistance $(R)$ and the overall thermal conductance of the module $(K)$ can also be obtained from literature [24]. In specific,

$$
\begin{aligned}
& R=N\left(\rho_{p} \frac{L_{p}}{A_{p}}+\rho_{n} \frac{L_{n}}{A_{n}}\right), \\
& K=N\left(k_{p} \frac{A_{p}}{L_{p}}+k_{n} \frac{A_{n}}{L_{n}}\right),
\end{aligned}
$$

where $\rho_{n}$ and $\rho_{p}$ are the electrical resistivities of the thermoelectric materials, $k_{n}$ and $k_{p}$ are the thermal conductivities, $A_{n}$ and $A_{p}$ are the cross-section areas of the thermoelectric pellets, and $L_{n}$ and $L_{p}$ are the lengths of the pellet.

Based on the equivalent circuit shown in Fig. 1(b), the current flowing through the load can be determined as

$$
I=\frac{V_{O C}}{R_{i n}+R_{L}}=\frac{V_{L}}{R_{L}},
$$

where $R_{i n}$ is the internal resistance of the TEG module, $R_{L}$ is the loading resistance, and $V_{L}$ is the loading voltage. $R_{\text {in }}$ can be expressed as

$$
R_{i n}=\frac{V_{O C}-V_{L}}{V_{L}} \times R_{L} .
$$

The power delivered to the load can be calculated by

$$
P=\frac{V_{O C}^{2}}{\left(R_{i n}+R_{L}\right)^{2}} \times R_{L} .
$$

These parameters will later be used to evaluate the power generation of the proposed thermal management system.

In this work, a commercially available $\mathrm{Bi}_{2} \mathrm{Te}_{3}$-based TEG is selected. The performance of TEG can be evaluated by a dimensionless quantity called the figure of merit $(Z T)$, which is defined as

$$
Z T=\frac{\alpha^{2} \sigma T}{k} .
$$

Since the Seebeck coefficients $\alpha$, electrical conductivity $\sigma$, and thermal conductivity $k$ of the p-type and n-type $\mathrm{Bi}_{2} \mathrm{Te}_{3}$ are all temperature dependent [25], the $Z T$ is also temperature dependent. According to the data given in literature [24], the $\alpha$, $\sigma$, and $k$ of the p-type and $\mathrm{n}$-type $\mathrm{Bi}_{2} \mathrm{Te}_{3}$ are expressed as functions of temperature $T$ in Table I. To have a clearer view, the variations of these parameters with the $T$ varying from 300 $\mathrm{K}$ to $700 \mathrm{~K}$ are plotted in Fig. 2. 
TABLE I

THE TEMPERATURE-DEPENDENT MATERIAL PROPERTIES OF THE TE LEGS

\begin{tabular}{llll}
\hline \hline Material & Seebeck coefficient $\alpha\left[\mathrm{VK}^{-1}\right]$ & Electrical conductivity $\sigma\left[\mathrm{Sm}^{-1}\right]$ & Thermal conductivity $k\left[\mathrm{Wm}^{-1} \mathrm{~K}^{-1}\right]$ \\
\hline $\mathrm{p}-\mathrm{Bi}_{2} \mathrm{Te}_{3}$ & $1.30 \times 10^{-9} \mathrm{~T}^{2}+1.17 \times 10^{-6} \mathrm{~T}-8.80 \times 10^{-5}$ & $1.80184 \mathrm{~T}^{2}-2101.88 \mathrm{~T}+686731.77$ & $3.11 \times 10^{-5} \mathrm{~T}^{2}-0.02413 \mathrm{~T}+5.90208$ \\
$\mathrm{n}-\mathrm{Bi}_{2} \mathrm{Te}_{3}$ & $-2.31 \times 10^{-9} \mathrm{~T}^{2}-1.65 \times 10^{-6} \mathrm{~T}+6.89 \times 10^{-5}$ & $0.89631 \mathrm{~T}^{2}-860.754 \mathrm{~T}+262853.95$ & $1.19 \times 10^{-5} \mathrm{~T}^{2}-0.00577 \mathrm{~T}+2.00418$ \\
\hline
\end{tabular}

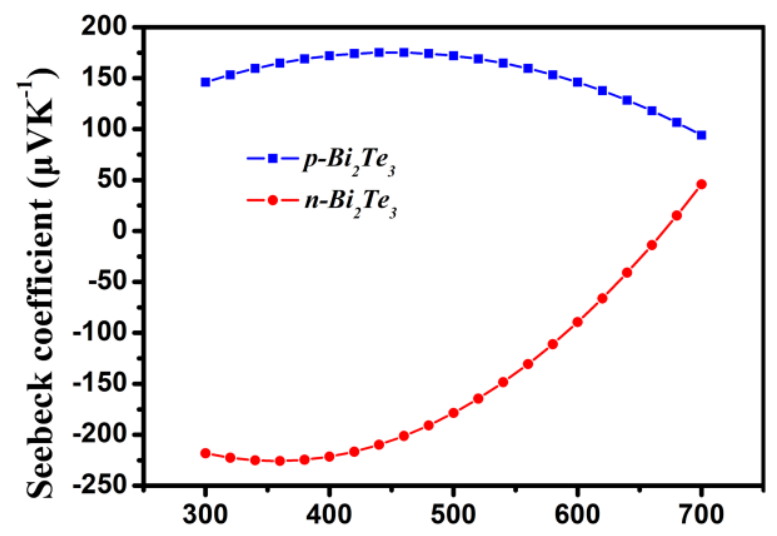

(a)

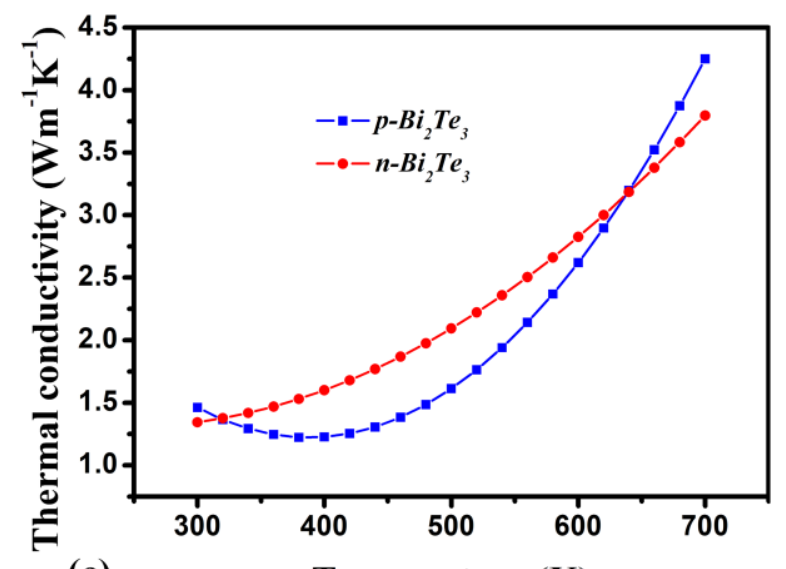

(c)
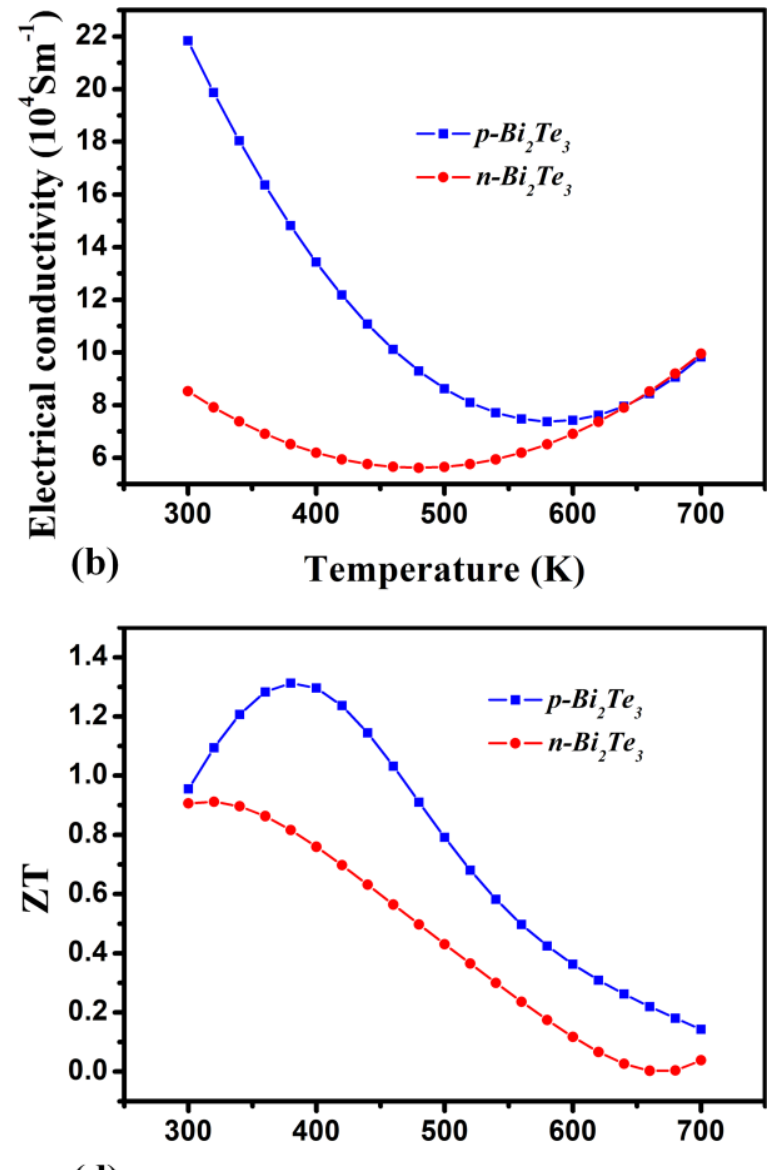

(d)

Temperature (K)

Fig. 2. Temperature-dependent thermoelectric properties of the n-type and p-type $\mathrm{Bi}_{2} \mathrm{Te}_{3}$ : (a) Seebeck coefficient $\alpha$; (b) electrical conductivity $\sigma$; (c) thermal conductivity $k$; (d) figure of merit $Z T$.

\section{B. Experiments on the output performance of TEG}

To investigate whether the selected $\mathrm{Bi}_{2} \mathrm{Te}_{3}$-based TEG can provide enough power for the sensor, as shown in Fig. 3(a), a power generation experimental platform is established to characterize the output performance of the TEG. The TEG is sandwiched between a constant-temperature heating platform and a heatsink with a fan to provide a temperature gradient between its two sides. Moreover, the contact surfaces of the TEG are coated with thermal grease to improve heat conduction. The hot-side and cold-side temperatures $T_{G H}$ and $T_{G C}$ of the TEG are monitored by an 8-channel temperature acquisition instrument (thermometer). The output voltage generated by the TEG is measured by a digital multimeter. Two K-type thermocouples probe are placed on the top and bottom layers of the TEG to acquire temperature. During the experiment, the indoor room temperature is maintained at $22^{\circ} \mathrm{C}$. The temperatures on the both sides of the TEG increase as the heating platform is gradually heated to $80^{\circ} \mathrm{C}$. However, $T_{G C}$ rises slower than $T_{G H}$ due to the heatsink and fan attached to the cold surface of the TEG.

Fig. 3(b) plots the variations of $V_{O C}$ and $V_{L}$ with $\Delta T(\Delta T=$ $T_{G H}-T_{G C}$ ). It can be seen from Fig. 3(b) that both $V_{O C}$ and $V_{L}$ increase almost linearly with $\Delta T$. Under the same $\Delta T, V_{O C}$ is always larger than $V_{L}$, which is expected from the equivalent circuit shown in Fig. 1(b). As $\Delta T$ varies from $0 \mathrm{~K}$ to $25 \mathrm{~K}, V_{O C}$ increases from $0 \mathrm{mV}$ to $558.68 \mathrm{mV}$. When $R_{L}=3 \Omega$, the value of $V_{O C}$ is approximately twice as large as $V_{L}$, which means that the voltages across $R_{L}$ and $R_{i n}$ are the same. Thus, from the circuit shown in Fig. 1(b), it can be easily deducted that $R_{i n} \approx R_{L}$ $=3 \Omega$.

Meanwhile, $R_{i n}$ can also be calculated by substituting the values of $V_{O C}, V_{L}$, and $R_{L}$ into (6). Fig. 3(c) plots the calculated $R_{i n}$ with $\Delta T$ changing from $0 \mathrm{~K}$ to $25 \mathrm{~K}$ when $R_{L}=1 \Omega, 2 \Omega$, and $3 \Omega$. According to the figure, the variations of $R_{\text {in }}$ is very small. Therefore, $R_{i n}$ is considered as a constant of $3.04 \Omega$ (average value) in this work for simplicity. 


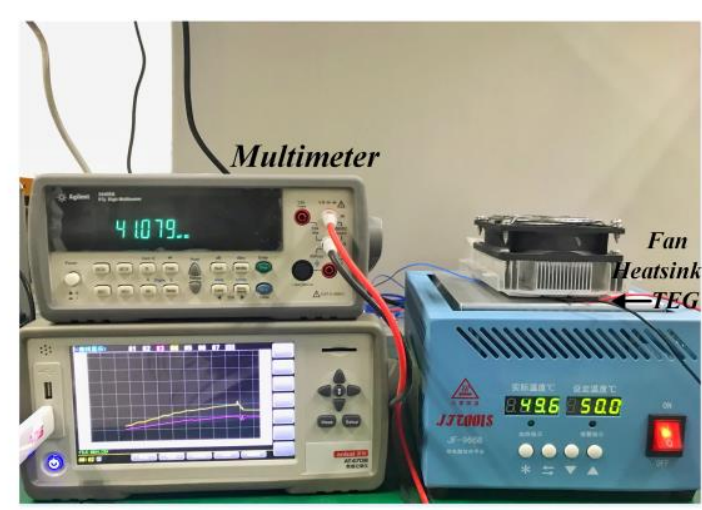

(a)

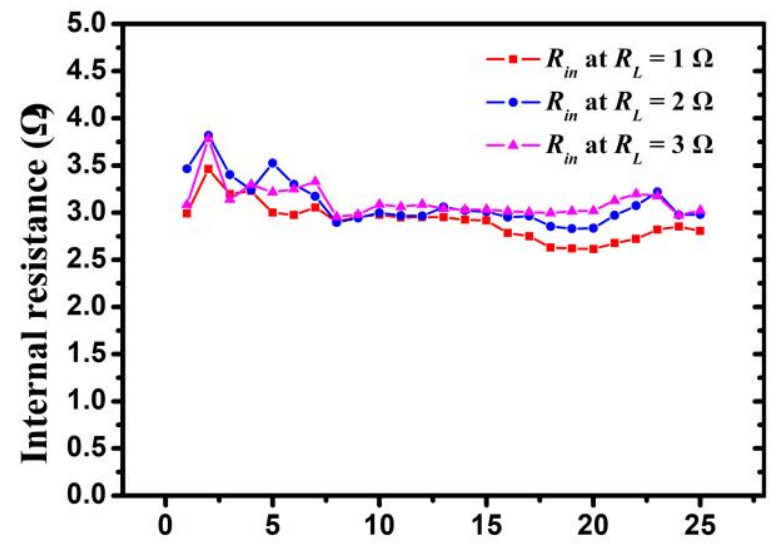

(c)

Temperature difference $(\mathrm{K})$

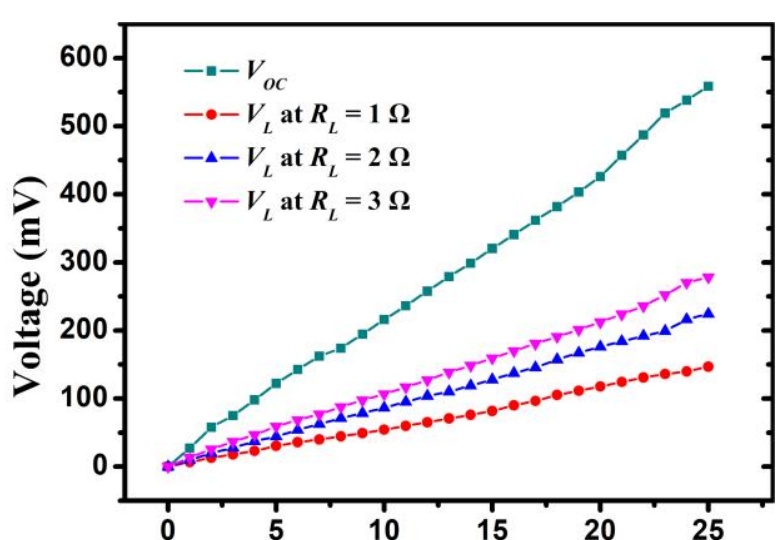

(b) Temperature difference (K)

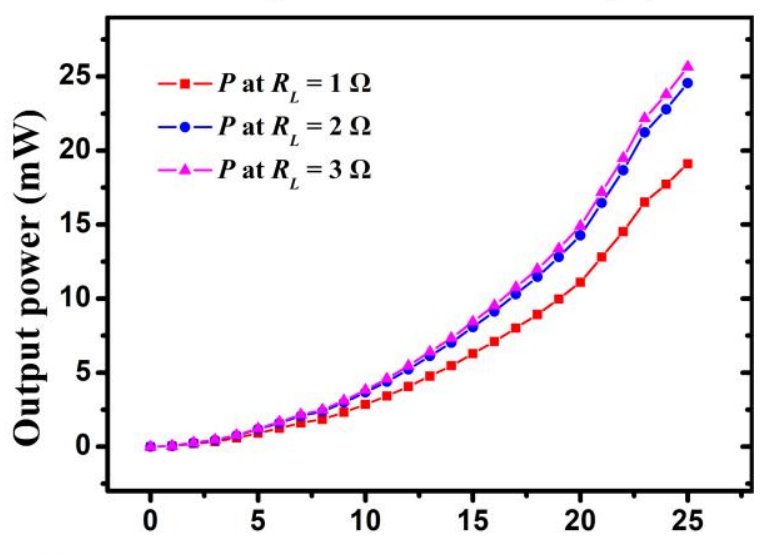

(d)

Temperature difference $(\mathrm{K})$

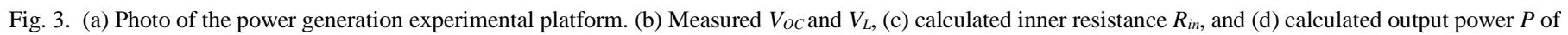
the TEG with different loading resistance $R_{L}$.

Subsequently, the output power $P$ generated by the TEG is calculated from (7) and is plotted in Fig. 3(d). According to Fig. $3(\mathrm{~d})$, the larger the $\Delta T$, the higher the $P$, which is in accordance with the expectation. On the other hand, the value of $R_{L}$ also affects $P$. According to the Thevenin's law, $P$ reaches the maximum value when $R_{L}=R_{i n}$. This explains why $P$ has larger values when $R_{L}=3 \Omega$ compared to the cases of $R_{L}=1 \Omega$ or $2 \Omega$. When $R_{L}=3 \Omega$, with $\Delta T$ changing from $0 \mathrm{~K}$ to $25 \mathrm{~K}, P$ is increased from $1.23 \mathrm{~mW}$ to $25.66 \mathrm{~mW}$. This milliwatt-level power is sufficient for many low-power devices.

In this section, the internal resistance $R_{\text {in }}$ of the TEG is determined. Both theory and experiment results show that, under different working conditions, $R_{i n}$ is quite stable and can be seen as a constant of $R_{\text {in }}=3.04 \Omega$.

\section{DESIGN OF THE THERMAL MANAGEMENT SYSTEM}

\section{A. Temperature acquisition module}

Temperature is the main factor influencing the photoelectric property of HP-LEDs [26]. Frequent overheating can cause unexpected shutdowns and shorten LED lifespans. If the surface temperature of working LEDs can be monitored, a protection module can be applied to prevent overheating.

As illustrated in Fig. 4 (a), a temperature acquisition module is established including a temperature sensor TMP116 [27], a microcontroller STM32 [28], and a host computer. In this work, the TMP116 from Texas Instruments is chosen to monitor and measure the surface temperature of a LED in real time. The communication protocol of TMP116 is compatible with the Inner-Integrated Circuit $\left(\mathrm{I}^{2} \mathrm{C}\right)$ bus interface. The static power consumption of TMP116 is very low most of the time, except for transmission bursts when the circuit is powered up to conduct measurements and transmit data. Typically, a current of $3.5 \mu \mathrm{A}$ is consumed by TMP116 in the operation voltage range of 1.9 to $5.5 \mathrm{~V}$. The STM32 microcontroller is programmed to communicate with the sensor via $\mathrm{I}^{2} \mathrm{C}$ bus. The data acquired by the temperature sensor is transmitted to and processed by STM32. Then, the processed data is further transmitted to the host computer through a serial interface. To visualize the temperature variations of the measured object, a software is developed with LabVIEW in the host computer, which can acquire, store, and display the temperature data, as illustrated in Fig. 4 (b).

The internal configuration register of TMP116 is initialized by STM32 so that the host computer can read the data. With the use of an oscilloscope, the waveform of the reading process is captured, as shown in Fig. 4 (c). The vertical grid is $2 \mathrm{~V}$ on the oscilloscope. According to the figure, the level of Serial clock (SCL) and Serial data (SDA) is approximately $3.2 \mathrm{~V}$ (it should be $3.3 \mathrm{~V}$ theoretically). The reading cycle is described as 
follows.

(1) First, the state of the SDA line is changed from high to low when the SCL line is at a high level, and the data transfer is initiated with a START condition. After that, a slave address (1001 0000) is sent from STM32 to TMP116. The last bit is 0, which is a writing signal. Then, an acknowledged bit (ACK) is generated by TMP116 for responding to the writing request.

(2) Second, the address of the temperature register $(0000$ 0000), which is to be read, is transmitted from STM32 to TMP116. Later, this register address is responded by TMP116, and a repeated START condition is sent via STM32. In subsequence, the slave address (1001 0001) is transmitted from STM32. The last bit is set to 1 , which signifies a reading. At this time, the reading request is acknowledged by TMP116, and the SDA bus is released through STM32.

(3) Finally, clock pulses are continuously sent out by STM32, followed by freeing the SDA line so that the data can be transferred from TMP116. At the end of the high byte of data (0000 1111), an ACK is sent through TMP116. When the low

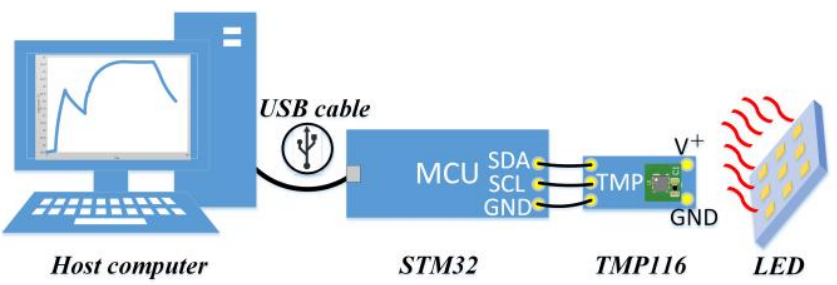

(a)

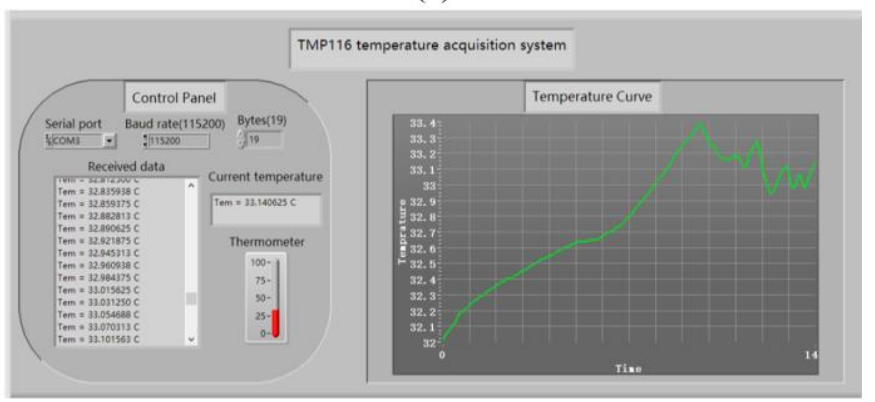

(b)

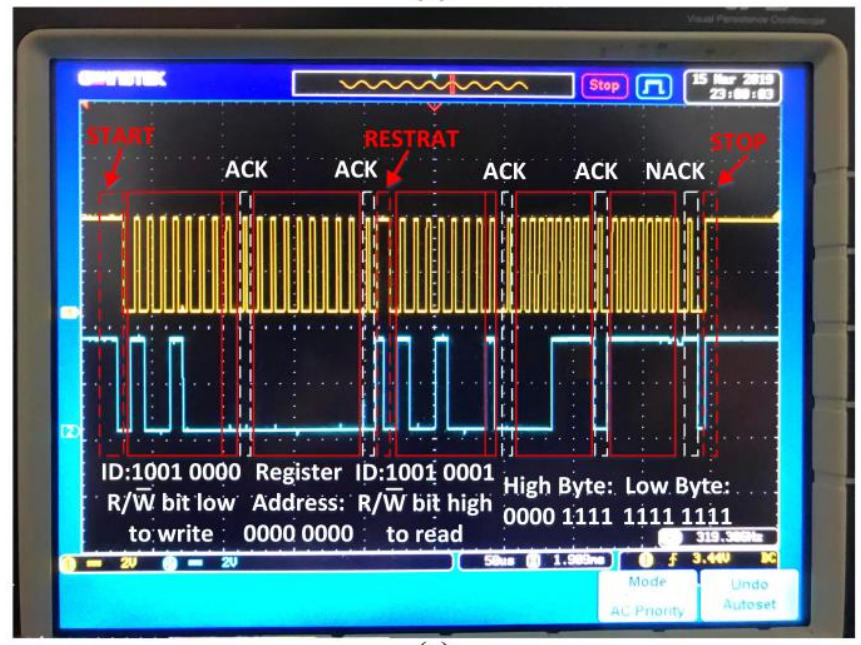

(c)

Fig. 4. The temperature acquisition model: (a) typical connected diagram, (b) the control panel of host computer based on LabVIEW, and (c) an oscilloscope capturing waveform of reading operation. bytes of data (1111 1111) are received by STM32, a non-acknowledged (NACK) signal is generated, which is a signal for halting communication and releasing the bus. Finally, data transfer is terminated with a STOP condition.

\section{B. DC-to-DC boost circuit}

The recommended operating voltage and power consumption for TMP116 are quite low, i.e., the minimal values of the voltage and power are $1.9 \mathrm{~V}$ and $6.65 \mu \mathrm{W}$, respectively. The study in Section II shows that the selected TEG can generate a much higher power than the power requested by TMP116. However, the output voltage of the TEG is in millivolt level, which is not enough to drive TMP116. For example, according to Figs. 3(b) and 3(d), with a $\Delta T$ of only 5 $\mathrm{K}$ and $R_{L}=3 \Omega$, the power $P \approx 2 \mathrm{~mW}(200 \mu \mathrm{W})$ and the output voltage $V_{L} \approx 50 \mathrm{mV}$. To power the temperature sensor, a higher voltage is required.

In this work, a DC-to-DC boost converter, i.e., LTC3108 [29], is used to raise the voltage generated by the TEG from millivolt level to volt level. The boost converter LTC3108 is configured as shown in Fig. 5. The input signal from VIN is first converted from a low DC voltage to an alternating-current (AC) voltage by a resonant boost oscillator, and then the charge is output from VOUT through the processing of internal rectifier and regulator. The voltage at the VOUT pin is regulated to the voltage selected by VS1 and VS2. Using jumper caps, VS1 is selected to connect with VAUX, while VS2 is tied to the ground in the circuit. By doing so, the voltage of the VOUT should be $3.3 \mathrm{~V}$ in theory, and this pin is chosen to supply power for the temperature sensors.

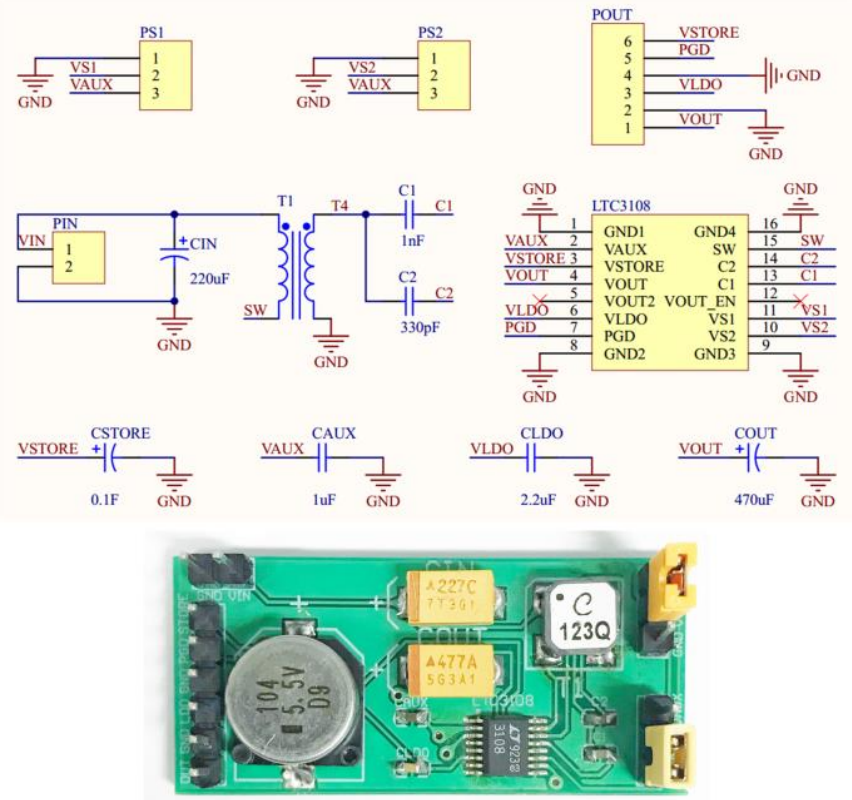

Fig. 5. LTC3108 boost circuit: schematic diagram and printed circuit board.

\section{Thermal management system}

With the temperature acquisition module and the DC-to-DC boost converter described in the previous subsections, the thermal management system is then developed. The physical 
layout and schematic electrical circuit of the system are shown in Figs. 6(a) and 6(b), respectively.

A white light LED with 9 light beads integrated inside is taken as the heat source in this system. The specifications of this LED are summarized in Table II. A TEG is sandwiched between the LED and a TEC with heatsink to obtain a thermal difference between its two sides. The power consumed by the TEC is $2.4 \mathrm{~V} \times 1.32 \mathrm{~A}=3.17 \mathrm{~W}$, which is provided by an external voltage source. The multifin heatsink is utilized as a heat exchanger to transfer the heat generated by the TEC, and a fan is screwed with the heatsink to blow the hot air away. Moreover, the back of the heatsink is covered by a 4.1-mm-thick shim, and all the contact surfaces between the LED, TEG, and TEC are coated with thermal grease to improve thermal conduction.

To monitor the surface temperature of the LED, a temperature sensor TMP116 is applied on top of the LED. The sensor is powered by the TEG harvesting the waste heat released from the LED. The data acquired by the sensor is transmitted to and displayed by a host computer through the microcontroller STM32. STM32 is powered by the host computer via a USB cable.

As presented in Fig. 6(b), a K-type thermometer is employed to monitor the surface temperature of the LED $\left(T_{L E D}\right)$, the cold-

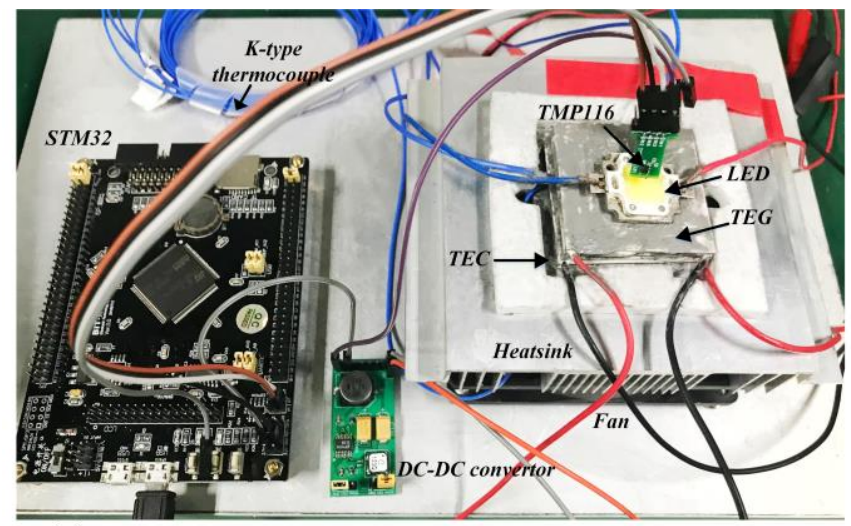

(a)

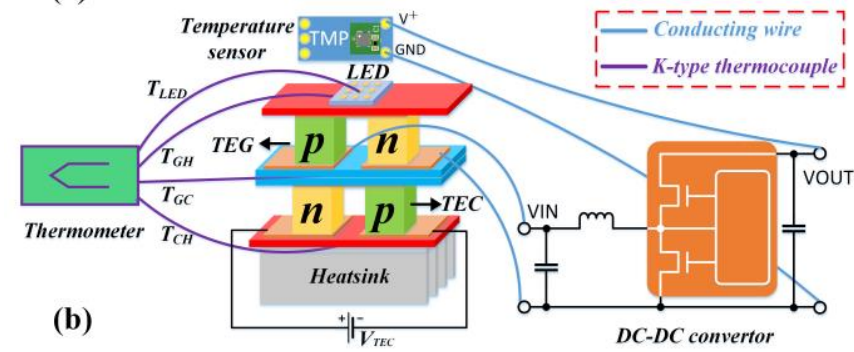

Fig. 6. The proposed thermal management system: (a) a physical map and (b) an electrical connected diagram.

TABLE II

PARAMETERS OF THE EMPLOYED HP-LED

\begin{tabular}{lll}
\hline \hline Parameter & Ratings & Units \\
\hline Chip size & $30 \times 30$ & $\mathrm{mil}^{2}$ \\
Operating voltage & 10 & $\mathrm{~V}$ \\
Current & 200 & $\mathrm{~mA}$ \\
Product size & $20 \times 29$ & $\mathrm{~mm}^{2}$ \\
Color temperature & White light $6000-7000$ & $\mathrm{~K}$ \\
\hline
\end{tabular}

and hot-side temperatures of the TEG, i.e., $T_{G C}$ and $T_{G H}$, and the cold- and hot-side temperatures of the TEC, i.e., $T_{C C}$ and $T_{C H}$. Note that $T_{G C}=T_{C C}$. In addition, a multimeter is used to measure the output voltage of the TEG.

\section{RESULTS AND DISCUSSION}

\section{A. Cooling performance evaluation}

Fig. 7(a) plots the variations of temperature at different test points during the experiment. The temperature data is acquired every 20 seconds. Fig. 7(a) shows that $T_{G C}$ decreases in the first time-interval from $0 \mathrm{~s}$ to $20 \mathrm{~s}$. This is due to the fact that the TEC's cooling starts immediately while it takes some time for the heat released from the LED to be transferred to the cold side of the TEC. From the second time-interval, heat is transferred to the cold side of TEC, whose cooling capacity is insufficient for cooling, and $T_{G C}$ begins to rise and gradually stabilize. Moreover, the other three temperatures $\left(T_{L E D}, T_{G H}\right.$, and $\left.T_{C H}\right)$ rise slowly in the beginning and then reach a balance after 540 seconds ( 9 minutes) of operation. This indicates that the system achieves a dynamic balance after a period of running time. Due to the presence of the LED and TEC, the top-side temperature of the TEG is higher than that at the bottom layer. In Fig. 7 (a), the final $T_{L E D}$ is approximately $32^{\circ} \mathrm{C}$, and the $\Delta T$ between $T_{G H}$ and $T_{G C}$ is $4.3 \mathrm{~K}$.

Moreover, a conventional TEC cooling system composed of TEC, heatsink, and fan is established and tested as a reference. The measured temperature data is plotted in Fig. 7(b). As observed from the figure, a dynamic balance is reached after approximately 120 seconds. It is noticed that the curves of $T_{L E D}$ and $T_{C C}$ are almost completely overlapped. They are stabilized at nearly $30^{\circ} \mathrm{C}$ when the dynamic balance is reached. In addition, Fig. 7(b) also includes the temperature variation of the LED without a cooling system, where $T_{L E D}$ increases rapidly from $22^{\circ} \mathrm{C}$ to $152^{\circ} \mathrm{C}$. This steep growth in temperature could endanger the operation of the LED. By comparing the results shown in Figs. 7(a) and 7(b), it can be concluded that the proposed thermal management system and the conventional cooling system have the similar cooling capability.

\section{B. Energy extraction from waste heat}

From the previous subsection, it is observed that the system generates a $\Delta T$ of $4.3 \mathrm{~K}$ when dynamic balance is achieved. This heat difference can be utilized and converted to electrical energy by the TEG and the DC-to-DC boost converter. The output voltage is measured by connecting the multimeter to the two terminals of the TEG in two cases. In the first case, the TEG is open loaded, thus the multimeter reads the output voltage $V_{O C}$. In the second case, the DC-to-DC boost converter and the temperature sensor are connected to the TEG, thus the multimeter measures the voltage across the load (DC-DC converter and sensor) $V_{L}$. The variations of $V_{O C}$ and $V_{L}$ with time are plotted in Fig. 7 (c). The output voltages $V_{O C}$ and $V_{L}$ are stabilized around $133.58 \mathrm{mV}$ and $115.19 \mathrm{mV}$, respectively. The attained $V_{O C}$ value is quite similar to the result shown in Fig. 3(b) when the TEG is assess alone, i.e., $V_{O C}$ is approximately $120 \mathrm{mV}$ when $\Delta T=4.3 \mathrm{~K}$. This shows that the TEG works 


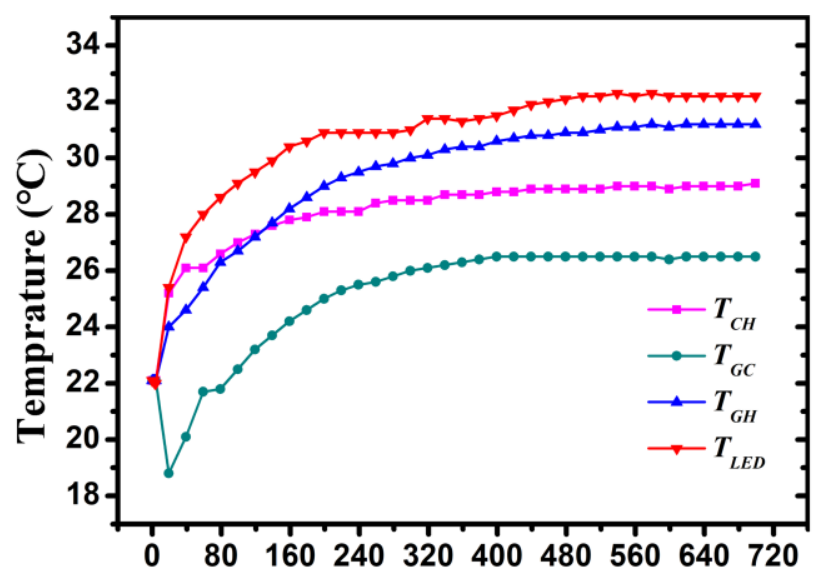

(a)

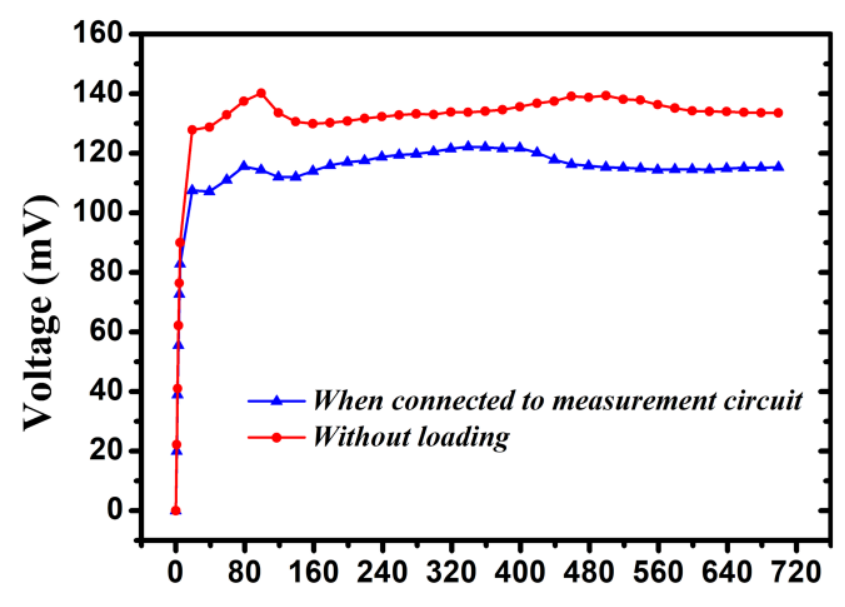

(c)
Time (s)

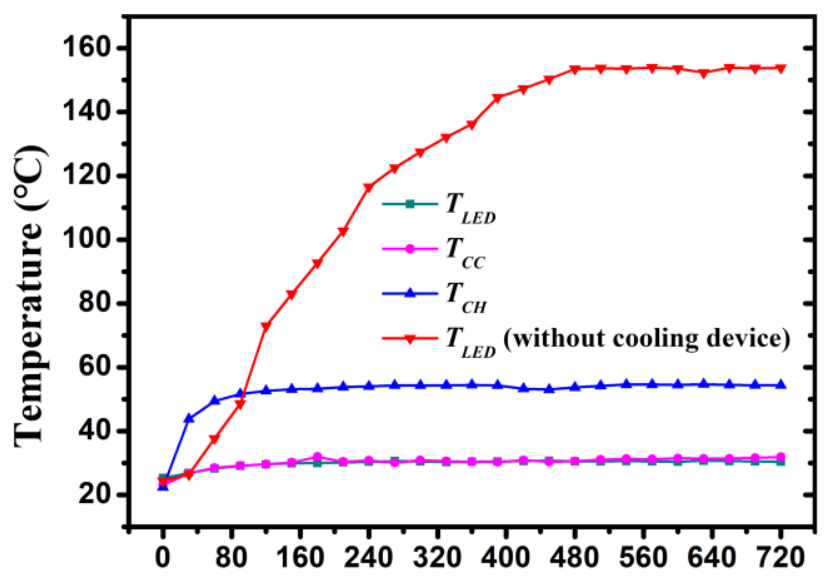

(b)

Time (s)

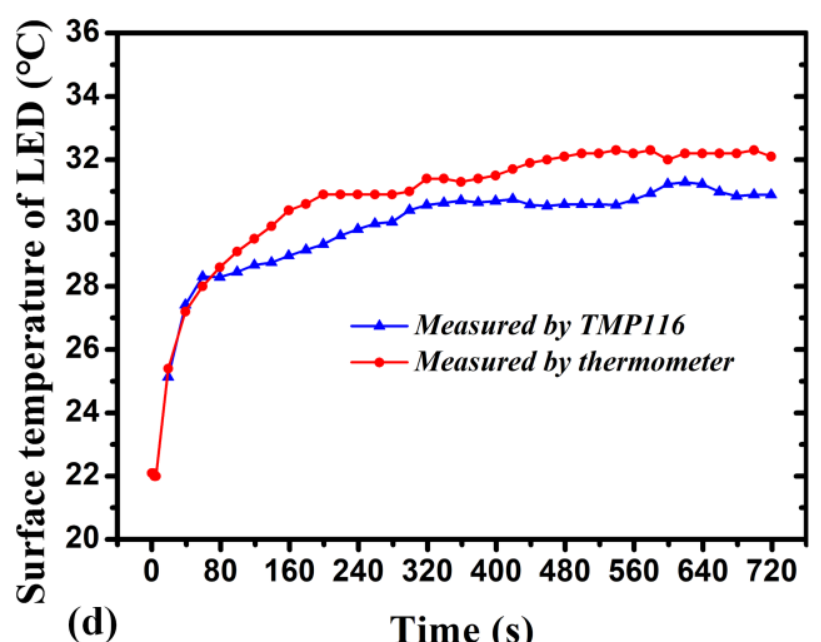

(d)

\section{Time (s)}

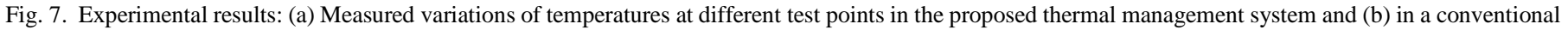

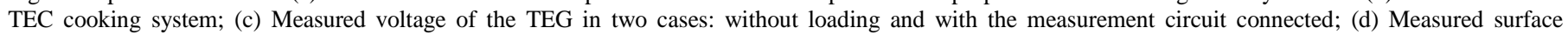
temperature of the LED by the thermometer and by the temperature sensor.

normally in the proposed thermal management system. The output voltage fluctuates in a small range, which illustrates that continuous electricity source is produced with almost no decline in power generation over the test period.

Considering the temperature measurement circuit acting as a resistive load, its equivalent resistance $\left(R_{e q}\right)$ can be calculated by

$$
R_{e q}=\frac{V_{L}}{V_{O C}-V_{L}} \times R_{i n} .
$$

By substituting the values of $V_{O C}, V_{L}$, and $R_{\text {in }}$ into (9), $R_{e q}$ is calculated to be $19.05 \Omega$. By substituting the calculated $R_{e q}$ into (7), the value of the power generation is obtained to be $P=$ $696.5 \mu \mathrm{W}$. Since the conversion efficiency of the LTC3108 boost circuit is approximately $55 \%$ according to its datasheet [29], the maximum $P$ of the LTC3108 is about $696.5 \mu \mathrm{W} \times 55 \%$ $=383 \mu \mathrm{W}$. This power is more than enough for the temperature sensor with its maximum power consumption is only $19.25 \mu \mathrm{W}$.

\section{Temperature monitoring with a temperature sensor}

The power harvested by the TEG allows the temperature sensor to work properly. It can monitor the surface temperature of the LED and the results are quite close to the results measured by the thermometer. The temperature data acquired by the sensor is compared with the temperature measured by the thermometer $\left(T_{K}\right)$ in Fig. $7(d)$. The results obtained from the sensor and the thermometer agree quite well with each other, i.e., the discrepancy between the two results is less than $5.38 \%$. With the employed sensor monitoring the temperature, a protection system can be developed to prevent overheating.

According to the measured results, the proposed system has a similar cooling capacity as the conventional TEC cooling system. Besides the cooling capability, it harvests thermal energy for power generation to drive a sensor for temperature monitoring. In the future, the thermal management system will be improved, harvesting more power that can be used for various purposes.

\section{CONCLUSION}

In this paper, a thermal management system that generates power from thermal waste released from HP-LEDs has been designed and validated. The proposed system can cool the LED as well as harvest energy from the LED. According to the measured results, the cooling capacity of the system is similar to the conventional TEC cooling system, i.e., the LED remains 
at $32^{\circ} \mathrm{C}$ when the room temperature is $22^{\circ} \mathrm{C}$. The power of the energy harvested from the $4.3 \mathrm{~K}$ thermal difference generated by the LED is approximately $696.5 \mu \mathrm{W}$, which can be used to power low-power electronics. In this work, the energy has been used to power a temperature sensor which only consumes 19.25 $\mu \mathrm{W}$ power as an example. With a DC-DC boost converter, $55 \%$ of the power can be converted with the output voltage increased to $3.3 \mathrm{~V}$ to power the sensor. The measurement results show that the temperature sensor works quite well. The discrepancy of the temperature measured by the sensor and that by the thermometer is less than $5.38 \%$. The proposed system demonstrates a way of recycling and reusing thermal waste from HP-LEDs.

\section{REFERENCES}

[1] Y. Zhang et al., "Analysis and Modeling of Thermal-Electric Coupling Effect of High-Power Monolithically Integrated Light-Emitting Diode," IEEE Trans. Electron Devices, vol. 65, no. 2, pp. 564-571, Feb. 2018.

[2] J. W. Mah, B. K. Lee, and M. Devarajan, "Thermal Impedance Measurement on Different Chip Arrangements for Various Multichip LEDs Application," IEEE Trans. Electron Devices, vol. 62, no. 9, pp. 2906-2912, Sep. 2015.

[3] D. M. K. Chakravarthii et al., "Thermal Characterization of LEDs Mounted on Substrates With Converging-Diverging and Diverging-Converging Channels," IEEE Trans. Electron Devices, vol. 64 no. 8, pp. 3308-3315, Aug. 2017.

[4] H. H. Cheng, D.-S. Huang, and M.-T. Lin, "Heat dissipation design and analysis of high power LED array using the finite element method," Microelectron. Reliab., vol. 52, no. 5, pp. 905-911, Jun. 2012.

[5] H. Chen, S. Lin, and C. Xiong, "Analysis and Modeling of Thermal Effect and Optical Characteristic of LED Systems With Parallel Plate-Fin Heatsink," IEEE Photonics J., vol. 9, no. 2, Apr. 2017.

[6] A. Christensen, and S. Graham, "Thermal effects in packaging high power light emitting diode arrays," Appl. Therm. Eng., vol. 29, no. 2-3, pp. 364-371, Mar. 2008.

[7] B. Ramos-Alvarado, B. Feng, and G. P. Peterson, "Comparison and optimization of single-phase liquid cooling devices for the heat dissipation of high-power LED arrays," Appl. Therm. Eng., vol. 59, pp. 648-659, Jun. 2013.

[8] H.-H. Wu, K.-H. Lin, and S.-T. Lin, "A study on the heat dissipation of high power multi-chip COB LEDs," Microelectron. J., vol. 43, no. 4, pp. 280-287, Feb. 2012.

[9] J. Zhou, X. Long, J. He, F. Ren, and L. Fang, "Uncertainty Quantification for Junction Temperature of Automotive LED With Die-Attach Layer Microstructure," IEEE Trans. Device Mater. Reliab., vol. 18, no. 1, pp. 86-96, Mar. 2018.

[10] C.-P. Wang, T.-T. Chen, H.-K. Fu, T.-L. Chang, P.-T. Chou, "Transient Analysis of Partial Thermal Characteristics of Multistructure Power LEDs," IEEE Trans. Electron Devices, vol. 60, no. 5, pp. 1668-1672, May. 2013.

[11] I. Y. Chen, M.-Z. Guo, K.-S. Yang, and C.-C. Wang, "Enhanced cooling for LED lighting using ionic wind," Int. J. Heat Mass Transfer, vol. 57, no. 1, pp. 285-291, Nov. 2013.

[12] N. Wang, X.-C. Li, and J.-F. Mao, "Improvement of Thermal Environment by Thermoelectric Coolers and Numerical Optimization of Thermal Performance," IEEE Trans. Electron Devices, vol. 62, no. 8, pp. 2579-2586, Aug. 2015.

[13] J. Wang, X.-J. Zhao, Y.-X. Cai, C. Z. Zhang, and W.-W. Bao, "Thermal Model Design and Analysis of High-Power LED Headlamp Cooling Device Based on the Thermoelectric Effect," IEEE Trans. Compon., Packag., Manuf. Technol., vol. 5, no. 5, pp. 641-649, May. 2015.

[14] W. Zhang, L. Shen, Y. Yang, and H. Chen, "Thermal management for a micro semiconductor laser based on thermoelectric cooling," Appl. Therm. Eng., vol. 90, pp. 664-673, Jul. 2015.

[15] N. Wang, H.-W. Li, C. Ding, L.-Y. Shi, H.-Z. Jia, Z.-D. Ren, and Z.-Y. Zhao, "A Double-Voltage-Controlled Effective Thermal Conductivity
Model of Graphene for Thermoelectric Cooling," IEEE Trans. Electron Devices, vol. 65, no. 3, pp. 1185-1191, Mar. 2018.

[16] J. Li, X. Zhang, C. Zhou, J. Zheng, D. Ge, and W. Zhu, "New Applications of an Automated System for High-Power LEDs," IEEE-ASME Trans. Mechatron., vol. 21, no. 2, pp. 1035-1042, Apr. 2016.

[17] S. U. Yuruker, E. Tamdogan, and M. Arik, "An Experimental and Computational Study on Efficiency of White LED Packages With a Thermocaloric Approach," IEEE Trans. Compon., Packag., Manuf. Technol., vol. 7, no. 2, pp. 201-207, Feb. 2017.

[18] S. Twaha, J. Zhu, Y. Yan, and B. Li, "A comprehensive review of thermoelectric technology: Materials, applications, modelling and performance improvement," Renew. Sust. Energ. Rev., vol. 65, pp. 698-726, Nov. 2016

[19] M. Lallart, L. V. Phung, and B. Massot, "Transformer-Free, Off-the-Shelf Electrical Interface for Low-Voltage DC Energy Harvesting," IEEE Trans. Ind. Electron., vol. 65, no. 7, pp. 5580-5589, Jul. 2018.

[20] Y. Wang, Y. Shi, D. Mei, Z. Chen, "Wearable thermoelectric generator to harvest body heat for powering a miniaturized accelerometer," Appl. Energy, vol. 215, pp. 690-698, Feb. 2018.

[21] A. Roy et al., "A $6.45 \mu \mathrm{W}$ Self-Powered SoC With Integrated Energy-Harvesting Power Management and ULP Asymmetric Radios for Portable Biomedical Systems," IEEE Trans. Biomed. Circuits. Syst., vol. 9, no. 6, pp. 862-874, Dec. 2015.

[22] P. C. Dias, et al., "Autonomous Multisensor System Powered by a Solar Thermoelectric Energy Harvester With Ultralow-Power Management Circuit," IEEE Trans. Instrum. Meas., vol. 64, no. 11, pp. 2918-2925, Nov. 2015.

[23] J. Chen, et al., "A Thermoelectric Energy Harvesting System for Powering Wireless Sensors in Nuclear Power Plants," IEEE Trans. Nucl. Sci., vol. 63, no .5, pp. 2738-2746, Oct. 2016.

[24] S. Qing, et al., "An Analytical Model for Performance Optimization of Thermoelectric Generator with Temperature Dependent Materials," IEEE Access, vol. 6, pp. 60852-60861, Nov. 2018.

[25] H. Sun, Y. Ge, W. Liu, and Z. Liu, "Geometric optimization of two-stage thermoelectric generator using genetic algorithms and thermodynamic analysis," Energy, pp. 37-48, Jan. 2019.

[26] H. Chen, F. Chen, S. Lin, and C. Xiong, "Thermal Analysis of a Multichip Light-Emitting Diode Device With Different Chip Arrays," IEEE Trans. Electron Devices, vol. 64, no. 12, pp. 5001-5005, Dec. 2017.

[27] TMP116 High-Accuracy, Low-Power, Digital Temperature Sensor with SMBus- and $I^{2} C$-Compatible Interface Datasheet, Texas Instruments., Dallas, TX, USA, 2017.

[28] High-Density Performance Line ARM-Based 32-Bit MCU with 256 to 512KB Flash, USB, CAN, 11 Timers, 3 ADCs, 13 Communication Interfaces Datasheet, STMicroelectronics., Geneva, CH, 2009.

[29] LTC3108 Ultralow Voltage Step-Up Converter and Power Manager Datasheet, Linear Technol., Milpitas, CA, USA, 2013. 